\title{
DEGRADACIÓN DEBIDO A LA ACCIÓN BIOLÓGICA DE EXCREMENTOS DE MURCIÉLAGOS EN LAS OBRAS DE ARTE ESPECIALES EN LAS CARRETERAS DE BRASIL
}

\author{
C. A. A. L. ANHAIA ${ }^{2}$, J. F. VIEIRA ${ }^{2}$, M. Y. F. CÂMARA ${ }^{3}$, B. E. SOBRINHO', \\ R. PEREIRA ${ }^{3}$, J. M. SARKIS ${ }^{3}$, A. V. PINTO JÚNIOR ${ }^{1}$, L. R. PERI ${ }^{1}$ \\ ${ }^{1}$ Departamento Nacional de Infraestrutura de Transportes (DNIT), Brasil. \\ ${ }^{2}$ Engefoto Engenharia e Aerolevantamentos SA \\ ${ }^{3}$ Strata Engenharia LTDA
}

\begin{abstract}
RESUMEN
Las obras de arte especiales (OAE's) son de gran importancia dentro del contexto de la red de carreteras, por lo que se requiere mayor cuidado cuando se trata con este tipo de estructura. Con los años, las OAE's comienzan a acortar su vida útil y comienzan a ocurrir degradaciones debido a acciones biológicas, químicas y físicas, que pueden contribuir a situaciones críticas de gran preocupación e incluso colapso. En este sentido, se abordará en las notas de trabajo sobre degradaciones biológicas, más específicamente las de los murciélagos guanos y sus características en relación con otras manifestaciones patológicas. Se utilizó un tramo de carretera del estado de Rio Grande do Norte, con alrededor de 40 OAE's, y datos del Sistema de Gestión de Obras Especiales (SGO), proporcionado por el Departamento Nacional de Infraestructura de Transporte (DNIT), y de estos datos, se realizó un estudio de caso. Los resultados indicaron que aproximadamente el $40 \%$ de los trabajos del tramo pudieron encontrar el daño causado por la secreción de los murciélagos, y esto ha causado un mayor deterioro del concreto. Con estos datos, fue posible concluir que la importancia de inspeccionar las obras, para garantizar la seguridad en las carreteras.
\end{abstract}

Palavras clave: Obras de arte especiales; puentes; degradación biológica; patologías; secreción de los murciélagos. 


\section{INTRODUCCIÓN}

Las Obras de arte especiales (OAE) son estructuras denominadas puentes, viaductos y pasarelas que requieren un proyecto de ejecución único. Estas estructuras están construidas para superar obstáculos como depresiones, cuerpos de agua, carreteras y ferrocarriles, y tienen una luz libre de más de seis metros. También es digno de mención que en este concepto se incluyen trabajos tales como pasos inferiores y superiores (DNIT 010-PRO, 2004).

Dentro de la red de carreteras, las OAE's se consideran obras expresivas y de gran interferencia, porque de acuerdo con las características de los relieves presentes en las carreteras, permiten el flujo, sin causar interrupciones en el sistema de transporte.

Las OEA son estructuras que están sujetas a acciones externas debido a cargas de movimiento del vehículo, fuerzas del viento, acciones del agua del río, variaciones de temperatura, contracción y fluencia en el caso de estructuras de concreto. Además, son obras construidas en entornos sujetos a diferentes clases de agresividad, desde lugares con agresividad leve hasta lugares con agresividad muy fuerte (ARAÚJO, 2014).

Debido a su funcionalidad, las OAE's pueden convertirse en puntos críticos en una carretera cuando sus condiciones de uso no son favorables, es decir, su integridad estructural no se mantiene en un nivel de uso adecuado. Las degradaciones de estas estructuras están vinculadas a las acciones y al entorno en el que se inserta, así como a la falta de actividades de mantenimiento de sus elementos componentes. Sin embargo, diferentes agentes externos, ya sean biológicos, físicos o químicos, contribuyen al aumento en el número de situaciones críticas encontradas en diferentes obras de arte especiales y pueden considerarse como focos potenciales para acelerar la degradación de los elementos.

Para ejemplificar casos de degradación biológica, podemos mencionar la presencia de escombros de construcción junto con desechos orgánicos, que se originan de la secreción de murciélagos en las estructuras, lo que provoca la aparición de humedad y desencadena otras patologías.

Para el debido cuidado de las OAE's, el Departamento Nacional de Infraestructura de Transporte (DNIT) ha desarrollado un estándar específico para la gestión, monitoreo e inspección de puentes, viaductos y túneles, que utiliza como fuente de referencia técnica primaria en las inspecciones realizadas en conjunto con la NBR 9452 (ABNT, 2016). A su vez, los datos de inspección se ingresan en el Sistema de Gestión de Obras Especiales (SGO), que implica el registro, la evaluación y el monitoreo de más de 6,000 estructuras ubicadas en las carreteras federales administradas por la agencia. En este contexto, este documento abordará las notas sobre la degradación en los OAE's debido a la presencia de agentes biológicos, más específicamente los de los murciélagos y sus características en relación con otras manifestaciones patológicas, que a menudo terminan confundiéndose con otras condiciones.

El objetivo de este estudio es evaluar una carretera en el estado de Rio Grande do Norte que contemple la ocurrencia de esta patología y proponer mejoras en los sistemas estructurales y el mantenimiento de estos trabajos con altas tasas de degradación, para contribuir al mejoramiento de la malla vial.

\section{INSPECCIONES EN LAS OBRAS DE ARTE ESPECIALES}

Las inspecciones son actividades que van desde la recopilación de información existente, en los diseños e información pertinente de las etapas de construcción y operación, hasta la evaluación del estado de la estructura y las recomendaciones apropiadas que se pueden realizar, que pueden ser re-inspecciones, trabajos de mantenimiento, trabajos de restauración, refuerzo o rehabilitación (DNIT, 2004).

Estas inspecciones tienen por objeto certificar la integridad estructural, seguridad y la comodidad del usuario durante desplazamiento en la autopista, así como extender la vida útil y mejorar el sistema de gestión para planificar y financiar el mantenimiento y rehabilitación de estructuras. Por lo tanto, es de fundamental importancia que los datos se recopilen de manera sistemática y de acuerdo con los 
procedimientos de los Manuales de Inspección de Obras Especiales (HORTO, 2000). Con este fin, la necesidad y la frecuencia de estas inspecciones se discuten cada vez más en la comunidad científica. En la mayoría de los casos, estos intervalos definen la disminución de los gastos por posibles reparaciones de gran preocupación.

La gran preocupación por la gestión de las OAE's alentó al DNIT a elaborar el Sistema de Gestión de Obras Especiales (SGO), que es una herramienta que permite a la agencia tomar decisiones para mantener el conjunto de obras en buen estado. Según el DNIT 010-PRO (2004), las inspecciones pueden clasificarse en: catastrales, rutinarias, extraordinarias, especiales e intermedias. Así, la Tabla 1 muestra la descripción y las frecuencias recomendadas en los estándares.

Tabla 1. Resumen de los tipos de inspecciones y las frecuencias según la norma DNIT 010 (2004)

\begin{tabular}{c|c|c}
\hline Tipo & Definición & Frecuencia \\
\hline Catastral & $\begin{array}{c}\text { Inspección realizada preferencial o } \\
\text { obligatoriamente después de la construcción de } \\
\text { la obra, servirá de referencia para todas las } \\
\text { inspecciones posteriores. }\end{array}$ & $\begin{array}{c}\text { Inmediatamente } \\
\text { después de finalizada } \\
\text { la obra. }\end{array}$ \\
\hline Rutinaria & $\begin{array}{c}\text { En estas inspecciones son verificadas } \\
\text { visualmente la evolución de los daños, } \\
\text { detectados en inspecciones anteriores y nuevas } \\
\text { ocurrencias. }\end{array}$ & Cada 2 años. \\
\hline Especiales & $\begin{array}{c}\text { Inspección no programada, solicitada para } \\
\text { evaluar un daño estructural excepcional, } \\
\text { causado por el hombre o por la naturaleza. }\end{array}$ & Cada 5 años. \\
\hline Intermediaria & $\begin{array}{c}\text { Inspeciones detalladas, utilizando } \\
\text { instrumentación de precisión. }\end{array}$ & $\begin{array}{c}\text { Cuando ocurrir un } \\
\text { grave accidente. } \\
\text { Recomendada para monitorear una deficiencia. } \\
\text { Cuando sea } \\
\text { recomendado por } \\
\text { inspecciones } \\
\text { anteriores. }\end{array}$ \\
\hline
\end{tabular}

La NBR 9452 (ABNT, 2016) ha estado proporcionando estandarización con respecto a identificación y evaluación de los elementos constitutivos de los OAE's, junto con el DNIT 010-PRO estándar (DNIT, 2004). Estas normas proporcionan parámetros para la clasificación en cuanto al estado de estabilidad y conservación, agrupación con subsidios para priorizar acciones con el objetivo de mantenimiento e intervención de las obras como la gravedad de patologías.

Una vez que se inspecciona la OAE, se obtiene una visión general de la estructura por daños, ya sea por acciones biológicas, químicas y/o físicas, y el siguiente paso es evaluar la estructura. Esta evaluación se realiza con el fin de cuantificar, diagnosticar, clasificar y establecer las medidas que deben tomarse para el mantenimiento (FONSECA, 2007). Las notas para la clasificación de los elementos estructurales se pueden describir según la Tabla 2. 
Tabla 2. Resumen para los atributos de las notas de evaluación en las OAE's conforme la norma

DNIT 010 - PRO (2004)

\begin{tabular}{|c|c|c|c|c|}
\hline $\begin{array}{l}\text { Nota } \\
\text { Técnica }\end{array}$ & $\begin{array}{c}\text { Daños en elemento/ } \\
\text { insuficiencia } \\
\text { estructural }\end{array}$ & $\begin{array}{l}\text { Acción } \\
\text { correctiva }\end{array}$ & $\begin{array}{l}\text { Condición de } \\
\text { estabilidad }\end{array}$ & $\begin{array}{c}\text { Clasificación das condiciones } \\
\text { del puente }\end{array}$ \\
\hline 5 & $\begin{array}{l}\text { No hay daños ni } \\
\text { insuficiencia. }\end{array}$ & - & Excelente & Sin problemas \\
\hline 4 & $\begin{array}{l}\text { Hay daños, mas no } \\
\text { hay señales de } \\
\text { insuficiencia } \\
\text { estructural. }\end{array}$ & $\begin{array}{l}\text { Servicios de } \\
\text { manutención. }\end{array}$ & Buena & Sin problemas importantes \\
\hline 3 & $\begin{array}{l}\text { Hay daños } \\
\text { generando } \\
\text { insuficiencia, mas } \\
\text { sin } \\
\text { comprometimiento } \\
\text { de la estabilidad de } \\
\text { la obra. }\end{array}$ & $\begin{array}{c}\text { La } \\
\text { recuperación } \\
\text { puede ser } \\
\text { postergada, } \\
\text { mas el } \\
\text { problema } \\
\text { debe } \\
\text { mantenerse en } \\
\text { observación. } \\
\end{array}$ & $\begin{array}{c}\text { Buena } \\
\text { aparentemente }\end{array}$ & $\begin{array}{l}\text { Potencialmente problemática. } \\
\text { En este caso debe acompañarse } \\
\text { la evolución patológica, para } \\
\text { disminuir los riesgos de un } \\
\text { agravamiento de la } \\
\text { insuficiencia estructural. }\end{array}$ \\
\hline 2 & $\begin{array}{l}\text { Daños con } \\
\text { significativa } \\
\text { insuficiencia en el } \\
\text { puente, por lo que } \\
\text { hay riesgo de } \\
\text { colapso estructural. }\end{array}$ & $\begin{array}{l}\text { La } \\
\text { recuperación } \\
\text { debe ser } \\
\text { realizada en } \\
\text { un plazo } \\
\text { corto. }\end{array}$ & Pobre & $\begin{array}{c}\text { Obra problemática } \\
\text { Demasiada demora puede } \\
\text { comprometer seriamente la vida } \\
\text { útil de la estructura. Se } \\
\text { recomiendan inspecciones } \\
\text { intermedias para el monitoreo } \\
\text { de enfermedades. }\end{array}$ \\
\hline 1 & $\begin{array}{l}\text { Hay daños } \\
\text { generando } \\
\text { insuficiencia grave, } \\
\text { en el elemento en } \\
\text { cuestión y se } \\
\text { encuentra en estado } \\
\text { crítico, habiendo un } \\
\text { riesgo de colapso } \\
\text { estructural. }\end{array}$ & $\begin{array}{l}\text { Recuperación } \\
\text { inmediata o } \\
\text { rápida } \\
\text { substitución } \\
\text { de la obra. }\end{array}$ & Precaria & $\begin{array}{c}\text { Obra crítica. } \\
\text { En algunos casos, configura } \\
\text { una situación de emergencia y } \\
\text { puede estar acompañado de } \\
\text { medidas preventivas especiales, } \\
\text { tales como: restricción de la } \\
\text { carga del puente, intervención } \\
\text { total o parcial del tráfico, } \\
\text { apuntalamiento provisional, } \\
\text { instrumentación con lecturas de } \\
\text { desplazamiento continuo, entre } \\
\text { otros. }\end{array}$ \\
\hline
\end{tabular}

Según el trabajo de Bastos y Miranda (2017), la degradación de las estructuras de concreto puede ser causada por factores físicos, químicos y biológicos. Entre los principales factores que contribuyen a la reducción de la vida útil de las estructuras se encuentran: grietas, averías, fallas en el sistema de drenaje, fallas en la carpeta de rodadura, fallas en el hormigonado, abrasión y corrosión.

La corrosión en el refuerzo de estructuras de hormigón armado es una de las más preocupantes, ya que afecta directamente la integridad estructural, además del alto costo de reparación (LIMA, 2005). Algunos factores como la porosidad, las grietas y la falta de cobertura son responsables de la oxidación del refuerzo cuando están expuestos por elementos agresivos (VITÓRIO, 2003). 
Un buen ejemplo de un agente agresor que causa corrosión en las OAE's se origina en las heces y la orina de los murciélagos, lo que conlleva a la presencia constante de humedad, provocando un deterioro temprano en el hormigón, los marcos de las vigas y losas (ANGELO, 2004).

\subsection{Degradación debido a la acción biológica}

La durabilidad de las estructuras de concreto debe enfocarse de manera sistémica e integral, involucrando varios tipos de conocimiento. También debe estimarse como resultado de las acciones tomadas en todas las etapas del proceso de construcción, desde la concepción hasta el uso de la estructura (MEDEIROS, et al. 2011). Sin embargo, la vida útil puede verse influenciada debido a varios factores, incluida la acción biológica.

El proceso de deterioro (químico y físico) a través de organismos vivos en estructuras de concreto se denomina comúnmente biodegradación y se puede clasificar en tres tipos: estético, físico y químico. Esta degradación por efecto químico consiste en la reducción de la vida útil de la estructura a través de cambios en la matriz y agregados cementosos, como resultado de las sustancias producidas por los agentes biológicos.

Wei y col. (2013) afirman que se ha desarrollado un número considerable de estudios centrados en la biodegradación de estructuras, principalmente debido a los costosos efectos de la biodegradación. Los organismos vivos aceleran los procesos de deterioro, que a menudo pueden ocurrir a velocidades más lentas a la falta de organismos vivos.

Los excrementos de los murciélagos pueden verificarse detectando manchas marrones o negras (aparentes) con gránulos, excrementos más viejos pueden ser de tonalidad gris. Estos excrementos pueden acumularse en el suelo, debajo de algún elemento del puente, o en componentes estructurales debajo de donde se posan los murciélagos (VDOT, 2010). Sus excrementos (guano) cuando están en contacto con el agua, son los principales responsables de la formación de sales que alteran el pH de la estructura, causando lixiviación, reacción iónica y expansión del refuerzo del desequilibrio (SIEDEL et al, 2008).

Según Steiger (2015), la biodegradación contribuye a la erosión de la superficie expuesta, reduciendo el grosor de la cubierta. Esto influirá significativamente en el aumento de la porosidad, facilitando el transporte de materiales agresivos a la matriz cementosa, lo que puede acelerar la aparición de grietas y, en consecuencia, el desprendimiento del hormigón. Estos pasos se pueden ejemplificar en la Figura 1.

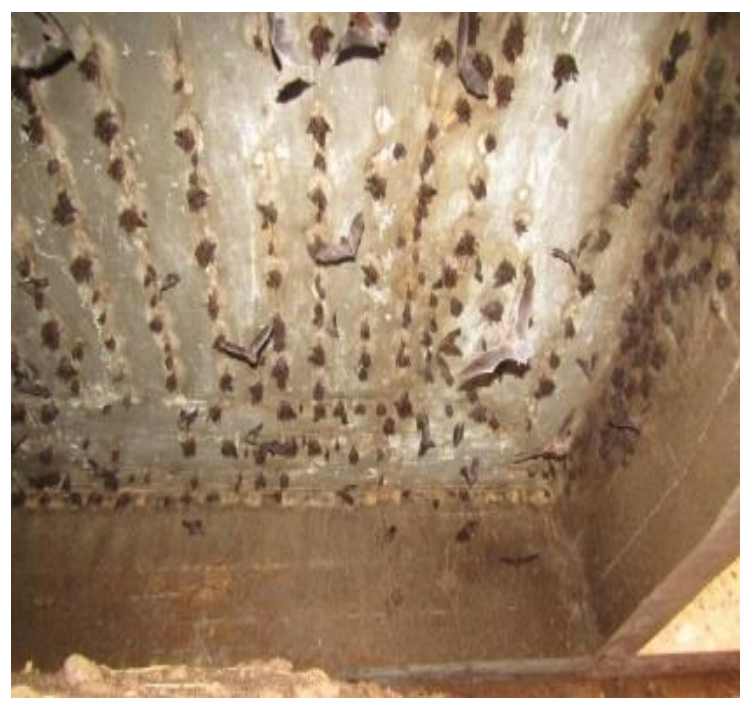

(a) Población de murciélagos en la estructura.

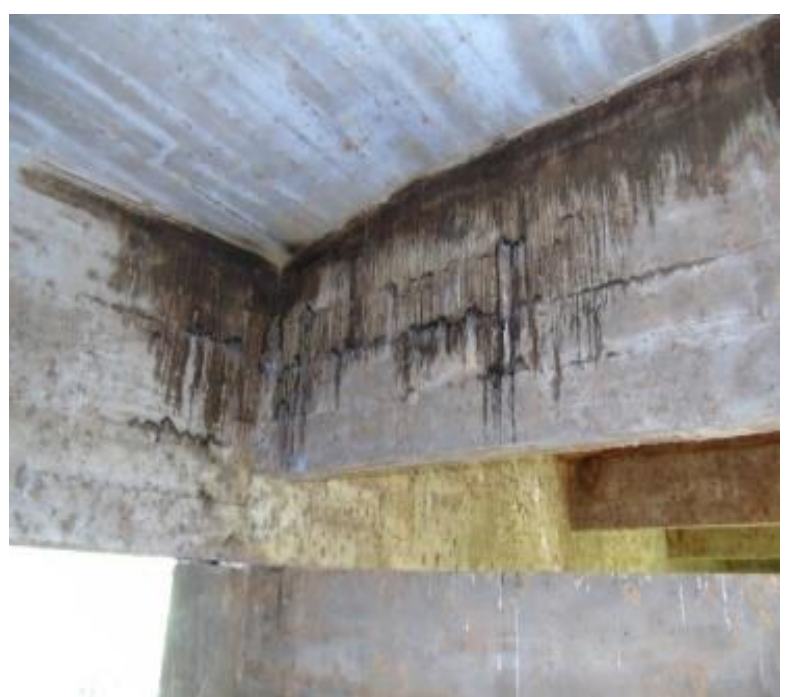

(b) Identificación de la fase inicial del daño con manchas marrones o negras en la Viga I de C.A. 


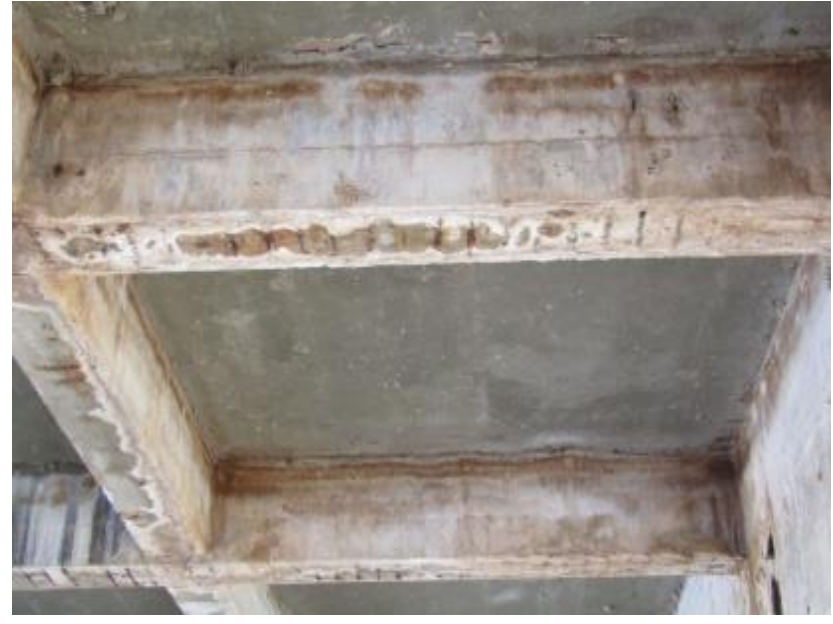

(c) Manchas blanquecinas debido a la evolución del daño.

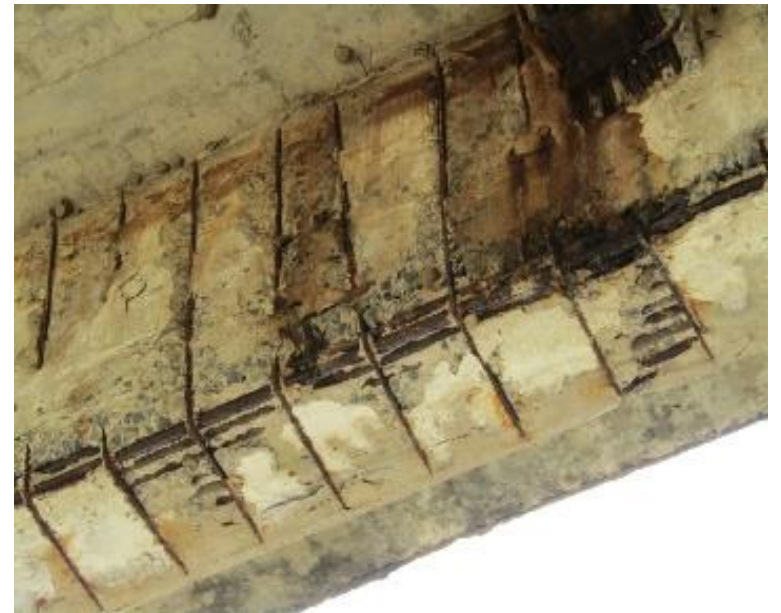

(d) Degradación en el hormigón debido a la acción biológica (guanos de murciélagos).

Figura 1. Evolución patológica de los elementos de una OAE debido a la acción de guano de murciélagos

Los factores más importantes para determinar la abundancia de murciélagos son la distribución y disponibilidad de alimentos y refugio. Con respecto al hábitat de estas especies, Bredt et al. (1998) afirman que incluyen lugares con condiciones ambientales favorables como temperatura ambiente, humedad relativa, poca luz y espacios no utilizados por personas.

Según la literatura, los entornos locales con llanuras de inundación fluviales, cursos de agua boscosos, setos y áreas de bosques antiguos, así como puentes, cobertizos y graneros están presentes en las áreas más habitadas por murciélagos (WSP, 2019) Las características como el tamaño de los refugios y su disponibilidad en un lugar determinado pueden interferir con el tamaño de las colonias y la aparición de la convivencia con otras especies (PACHECO et al. 2010).

Además de la falta de publicaciones considerables que involucren análisis de la degradación biológica presente en el contexto de los procedimientos de inspección de las OAE's de carreteras, existe una preocupación del Departamento Nacional de Infraestructura de Transporte (DNIT) en sus rutinas de inspección para considerar esta condición.

Al principio, el registro de la aparición de estos agentes externos, incentivará estudios y preocupaciones sobre estas condiciones. A partir de esto, se identifican los factores que contribuyen a acelerar los procesos de degradación, lo que hace que la gestión sea esencial para planificar propuestas de mantenimiento en OAE's.

\subsubsection{Murciélagos brasileños}

El libro "Murciélagos de Brasil" de Reis et al. (2007) afirma que en Brasil los murciélagos se llaman Andirá, Guandira o Guandiruçu en el idioma tupí. La falta de conocimiento sobre estos animales no se limita a estos nombres, sino a la diversidad de especies, la complejidad biológica y la importancia ecológica, como se muestra en la Figura 2. 


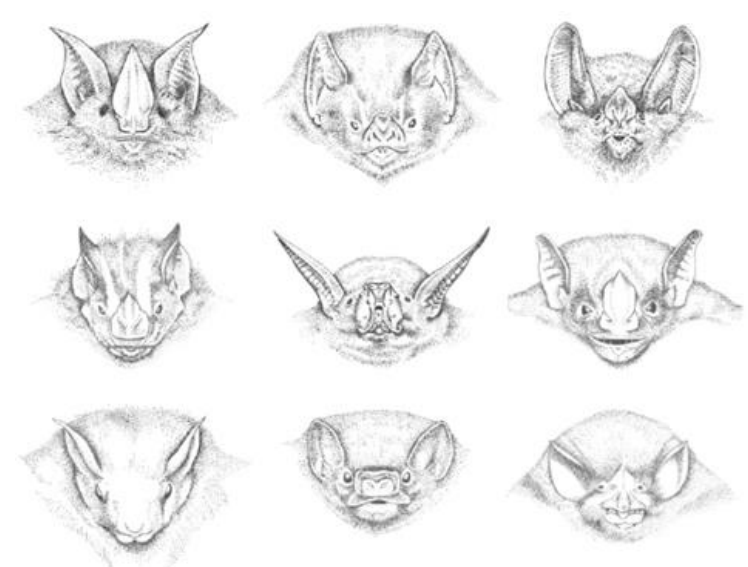

Figura 2. Demostración de la diversidad de especies de murciélagos en Brasil.

Este libro enfatiza que la diversidad de murciélagos de Brasil es una de las más grandes del mundo, y se encuentran alrededor de nueve familias, 64 géneros y 167 especies. Chiroptera es el segundo orden en riqueza que contiene más especies en todo el mundo, totalizando alrededor de 1200, siendo superado por Rodentia, con 2,277 especies (REIS, et al. 2007).

La colonia de murciélagos naturales más grande se encuentra en Bracken Bat, Texas, hogar de 20 millones de murciélagos, y la colonia urbana más grande de estas criaturas se encuentra en Austin, Texas, donde viven aproximadamente 1,5 millones de murciélagos. debajo del puente Ann W. Richards Congress Avenue, exactamente en una Obra de Arte Especial (REIS, et al. 2007).

En términos del hábitat preferido de estos animales son las grietas, grietas en las paredes de roca, la tala de árboles, los áticos y debajo de los puentes, que constituyen entornos que finalmente se convirtieron en uno de los más propicios para el desarrollo de estas poblaciones de mamíferos voladores. Durante el día, están protegidos por las OAE's y pueden ejercer sus hábitos nocturnos, en los que tanto la nariz como los oídos les permiten emitir y mejorar el ultrasonido, encontrar comida y ubicarse.

\subsubsection{Procedimiento de Inspección}

Conforme el Manual de Inspecciones para Obras de Arte Especiales en el punto 12.4.4 que trata sobre los "Procedimientos de inspección", Las vigas tipo cajón deben inspeccionarse cuidadosamente por fuera y por dentro; En el interior, las dificultades son mucho mayores debido a la escasa iluminación, la oxigenación del aire e incluso la posible existencia de gases tóxicos o explosivos, arácnidos y murciélagos.

No solo en la viga del ataúd se identifica la presencia de murciélagos, sino principalmente en los sistemas comunes dentro de la malla de carreteras, como aquellos con múltiples vigas largueras. El procedimiento de inspección se realiza según la Figura 3 a continuación.

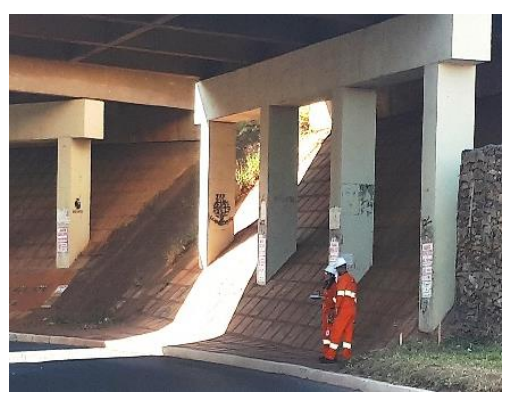

(a) inspección

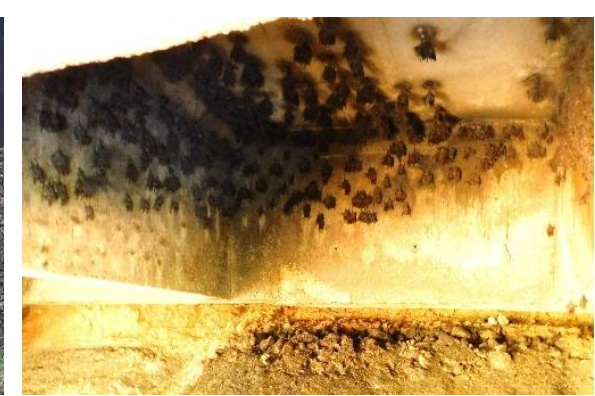

(b) identificación

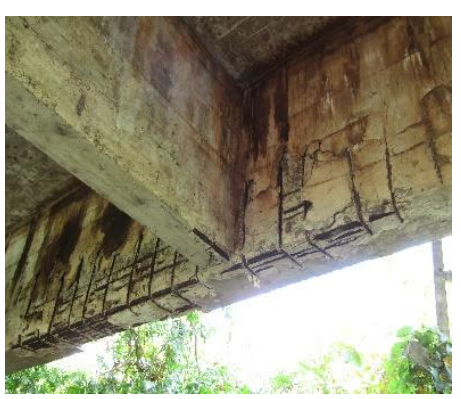

(c) patología

Figura 3. Procedimientos de inspección envolviendo la identificación de murciélagos en las regiones inferiores de las OAE's en las carreteras de Brasil. 
Estas patologías se vuelven extremadamente comunes en la inspección de OAE’s, siempre presentando el monitoreo y sus influencias en el análisis de las condiciones de estas importantes estructuras de carreteras.

\section{METODOLOGÍA}

El Departamento Nacional de Infraestructura y Transporte (DNIT) es responsable de la administración de más de 6,000 OAE's, sin incluir en este número los túneles y las vías peatonales. Para la gestión de este patrimonio utilizamos el Sistema de Gestión de Obras Especiales, que recibió el acrónimo SGO, y donde se tomarán los datos para su uso en esta investigación.

El procedimiento que se realizará en el trabajo se describe en 4 pasos, que se muestran en la Figura 4.

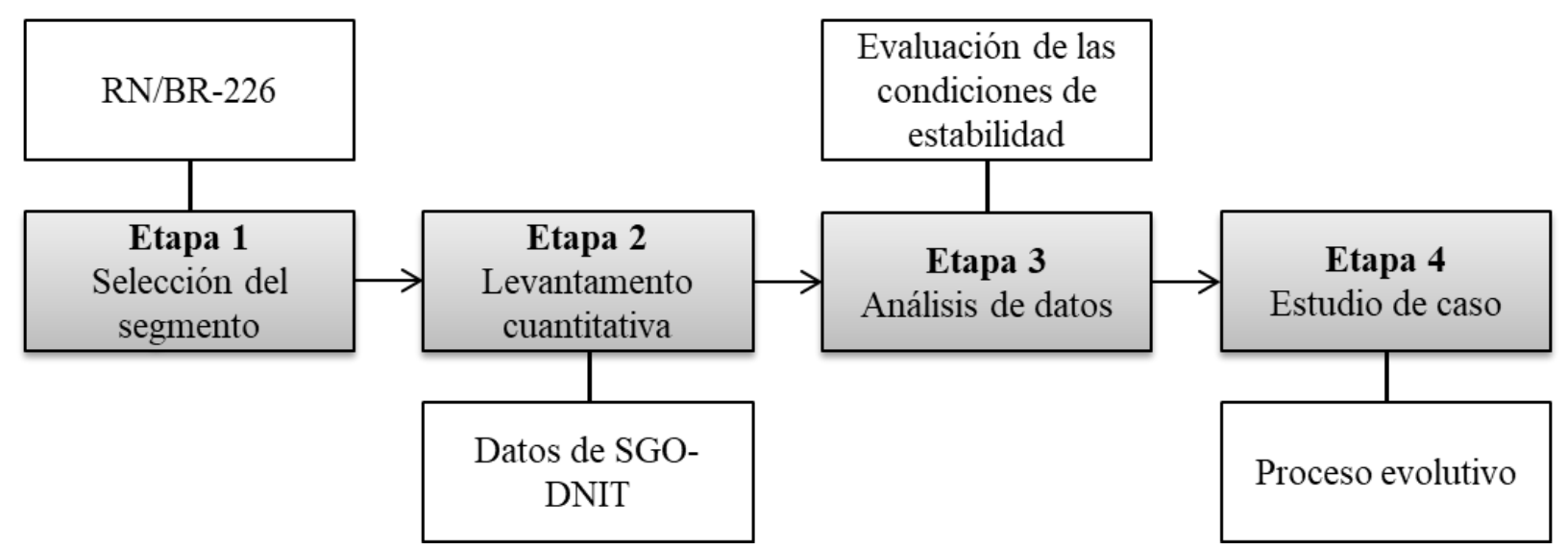

Figura 4. Secuencia de desarrollo de las etapas de este trabajo

En el Paso 1, buscamos un segmento con una alta incidencia de patología dentro del SGO y, por lo tanto, consideramos como objeto de estudio los OAE's presentes en la autopista BR-226, en el estado de Rio Grande do Norte (Figura 5). En total, se identificaron 43 obras registradas.

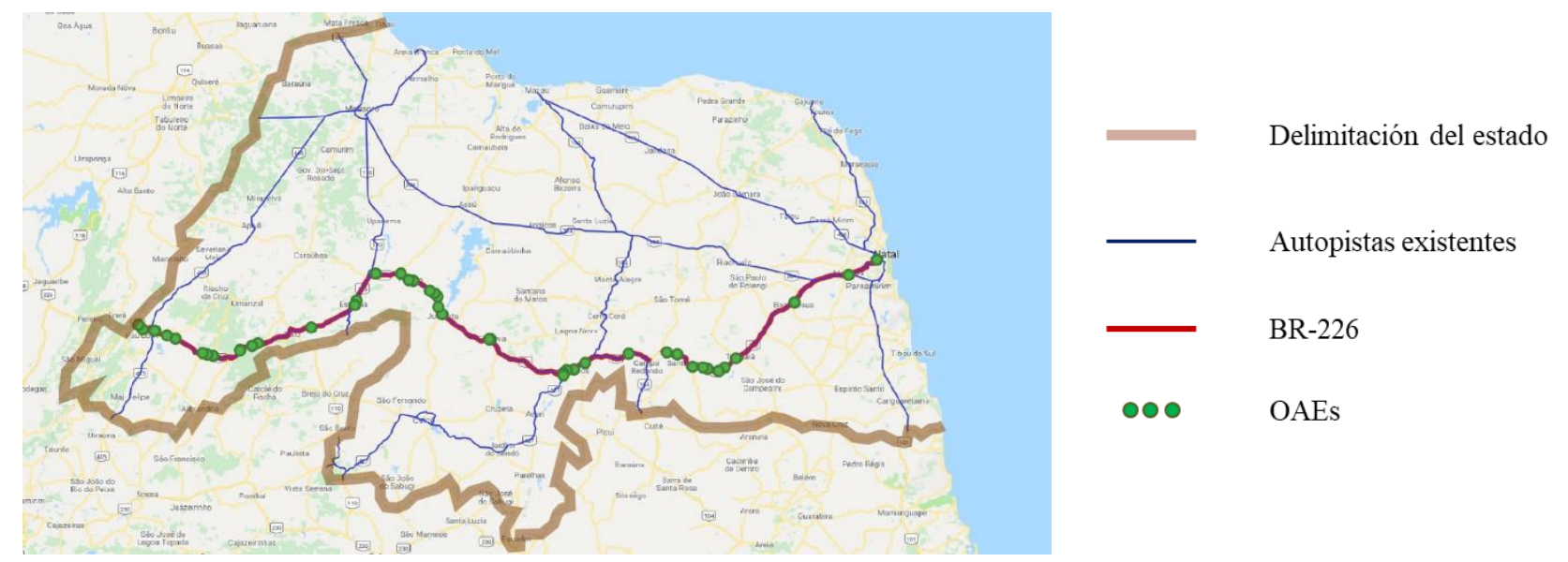

Figura 5. Localización de las OAE's en la autopista BR-226/RN

En el Paso 2 buscamos cuantificar las OAE's con manifestaciones patológicas causadas por la acción biológica de los murciélagos, haciendo una comparación con todas las OAE's presentes en el tramo elegido.

En la Etapa 3, se evaluó el estado de conservación de las OAE's degradadas debido a la acción biológica y la influencia del daño en las condiciones de estabilidad de la OAE's, de acuerdo con la nota técnica 
generada en la inspección. La nota corresponde a la condición de estabilidad del puente, recibiendo la nota del elemento estructural con menor nota de la OAE.

Por lo tanto, el Paso 4 constituye una investigación dentro de la base de datos, en la que se eligió el reporte con la mayor intensidad de daño, para realizar un estudio de caso. Este paso se realizó para verificar la evolución del daño y la degradación del elemento a partir de los datos de inspecciones anteriores realizadas hace 4 años en comparación con la inspección actual.

\section{RESULTADOS CUANTITATIVOS DEL LEVANTAMIENTO EN LAS OBRAS DE ARTE ESPECIALES POR EL DNIT}

Del objeto de estudio definido en la metodología de este trabajo, se identificaron 43 trabajos en la autopista BR-226, en Rio Grande do Norte. La Tabla 3 enumera las obras, su ubicación en la carretera $(\mathrm{km})$, el tipo de estructura principal y la presencia o ausencia de daños a los elementos principales.

Tabla 3: Resumen de las OAE's que presentan daños debido a la acción biodegradación biológica de los murciélagos en autopista BR-226/RN

\begin{tabular}{|c|c|c|c|c|c|}
\hline Identificación da OAE & km & SNV & $\mathbf{L}(\mathbf{m})$ & Daño & $\begin{array}{l}\text { Tipo de } \\
\text { estructura }\end{array}$ \\
\hline Puente km 0,35 & 0,35 & 226BRN0010 & 17,50 & si & Viga \\
\hline Puente sobre o Rio Jundiaí & 17,44 & 226BRN0030 & 31,00 & si & Viga \\
\hline Puente sobre o Rio Bom Jesus & 47,00 & 226BRN0070 & 17,50 & si & Viga \\
\hline Puente km 85,51 & 85,51 & 226BRN0130 & 19,50 & si & Viga \\
\hline Puente sobre o Riacho da Chapada & 92,21 & 226BRN0130 & 11,00 & si & Viga \\
\hline Ponton km 95,29 & 95,29 & 226BRN0130 & 5,70 & si & Viga \\
\hline Puente sobre o Riacho Logradouro & 100,78 & 226BRN0130 & 6,80 & si & Viga \\
\hline Puente km 103,10 & 103,10 & 226BRN0130 & 7,00 & no & Losa \\
\hline Puente sobre o Rio Inharé & 107,89 & 226BRN0150 & 40,00 & si & Viga \\
\hline Puente km 117,430 & 117,43 & 226BRN0165 & 11,10 & si & Viga \\
\hline Puente sobre o Riacho Bento Nunes & 130,05 & 226BRN0165 & 11,10 & si & Viga \\
\hline Puente km 143,80 & 143,80 & 226BRN0175 & 20,00 & no & Viga \\
\hline Puente km 165,63 & 165,63 & 226BRN0180 & 40,00 & no & Losa \\
\hline Puente sobre o Riacho Suçuarana & 171,66 & 226BRN0180 & 20,00 & si & Viga \\
\hline Puente sobre o Rio Currais Novos & 175,13 & 226BRN0180 & 64,30 & si & Viga \\
\hline Puente km 177,12 & 177,12 & 226BRN0200 & 33,00 & no & Viga \\
\hline Puente sobre o Riacho Frei Damião & 178,10 & 226BRN0210 & 23,00 & si & Viga \\
\hline Puente sobre o Riacho Russinho & 218,66 & 226BRN0220 & 39,50 & no & Viga \\
\hline Ponton km 219,67 & 219,67 & 226BRN0250 & 5,20 & no & Losa \\
\hline Puente sobre o Rio Piranhas - Açu & 245,66 & 226BRN0290 & 352,00 & no & Viga \\
\hline Puente Sobre Riacho Riachão & 250,11 & 226BRN0290 & 19,70 & si & Viga \\
\hline Puente Sobre Riacho do Alemão & 254,84 & 226BRN0290 & 16,00 & no & Losa \\
\hline Puente $\mathrm{km} \mathrm{257,08}$ & 257,08 & 226BRN0290 & 31,50 & no & Viga \\
\hline Puente sobre o Riacho do Vital & 259,58 & 226BRN0290 & 32,40 & no & Losa \\
\hline Puente sobre o Riacho do Areré & 268,22 & 226BRN0290 & 32,50 & no & Losa \\
\hline Puente km 270,21 & 270,21 & 226BRN0290 & 32,50 & no & Losa \\
\hline Puente Sobre o Riacho do Tapuio & 275,37 & 226BRN0290 & 48,10 & no & Losa \\
\hline Puente km 287,48 & 287,48 & 226BRN0300 & 20,60 & si & Viga \\
\hline Puente sobre o Riacho das Cacimbas & 303,19 & 226BRN0305 & 10,90 & si & Viga \\
\hline Puente sobre o Riacho do Livramento & 305,61 & 226BRN0305 & 21,90 & si & Viga \\
\hline
\end{tabular}




\begin{tabular}{|c|c|c|c|c|c|}
\hline Identificación da OAE & km & SNV & $\mathbf{L}(\mathbf{m})$ & Daño & $\begin{array}{l}\text { Tipo de } \\
\text { estructura }\end{array}$ \\
\hline Puente sobre o Riacho Cacimba de Baixo & 328,29 & 226BRN0315 & 13,00 & no & Losa \\
\hline Puente km 355,45 & 355,45 & 226BRN0363 & 32,50 & no & Losa \\
\hline Puente km 358,64 & 358,64 & 226BRN0366 & 35,00 & no & Losa \\
\hline Puente km 364,51 & 364,51 & 226BRN0370 & 32,50 & no & Losa \\
\hline Puente km 364,92 & 364,92 & 226BRN0370 & 16,00 & no & Losa \\
\hline Puente km 379,27 & 379,27 & 226BRN0377 & 32,50 & no & Losa \\
\hline Puente km 382,25 & 382,25 & 226BRN0380 & 6,00 & no & Losa \\
\hline Puente km 384,35 & 384,35 & 226BRN0380 & 32,80 & no & Losa \\
\hline Puente km 398,96 & 398,96 & 226BRN0380 & 16,00 & no & Losa \\
\hline Ponton km 402,99 & 402,99 & 226BRN0380 & 5,80 & no & Losa \\
\hline Puente sobre o Rio Apodi & 409,12 & 226BRN0380 & 190,00 & no & Viga \\
\hline Puente sobre o Rio Cachoeirinha & 416,70 & 226BRN0400 & 49,00 & no & Losa \\
\hline Puente sobre o Riacho do Cabelo & 419,40 & 226BRN0400 & 70,40 & no & Losa \\
\hline
\end{tabular}

En la Tabla 3 fue posible identificar que todas las obras que contienen el daño corresponden a puentes, como se esperaba, debido a las características del entorno en el que se insertan. Extrayendo los datos de la Tabla 3, se cuantificó el porcentaje de trabajos que presentaron el daño por degradación por acción biológica, que se presenta en la Figura 6.

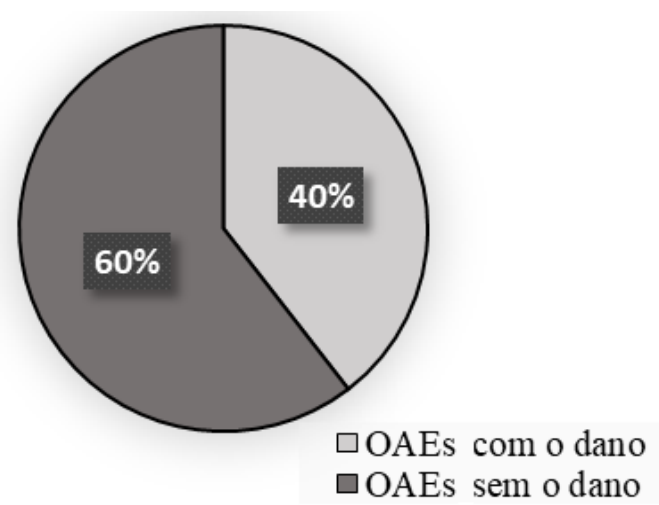

Figura 6. Porcentaje de OAE's con o sin manifestación patológica debido a biodegradación por acción de excrementos de murciélagos

La Figura 6 muestra que el 40\% de las OEA de la autopista en estudio tienen alguna manifestación patológica debido a excrementos de murciélago. En número, se identificaron 17 obras con el daño, entre SNV 226BRN0010 y 226BRN0305, con un total de aproximadamente $420 \mathrm{~km}$ de longitud. Entre los trabajos analizados, se verificó el tipo de estructura y se notó que todas las ocurrencias de daños se encuentran en estructuras cuyo elemento principal son las vigas T o I de hormigón armado. Las otras estructuras identificadas en el tramo tienen el tipo de estructura clasificada como puente losa, y en estos, el daño no fue identificado. Esto puede explicarse por el hecho de que los murciélagos proliferan en lugares menos brillantes, como los espacios internos entre vigas y losas.

Después de identificar el daño a los elementos del puente, se evaluaron los grados otorgados a los elementos estructurales, que se muestran en la Figura 7. Esta figura muestra la influencia del daño en el acortamiento de la vida útil de la estructura, en la que se clasificó aproximadamente el $65 \%$. con grado 2 (problemático), según datos de la SGO. 


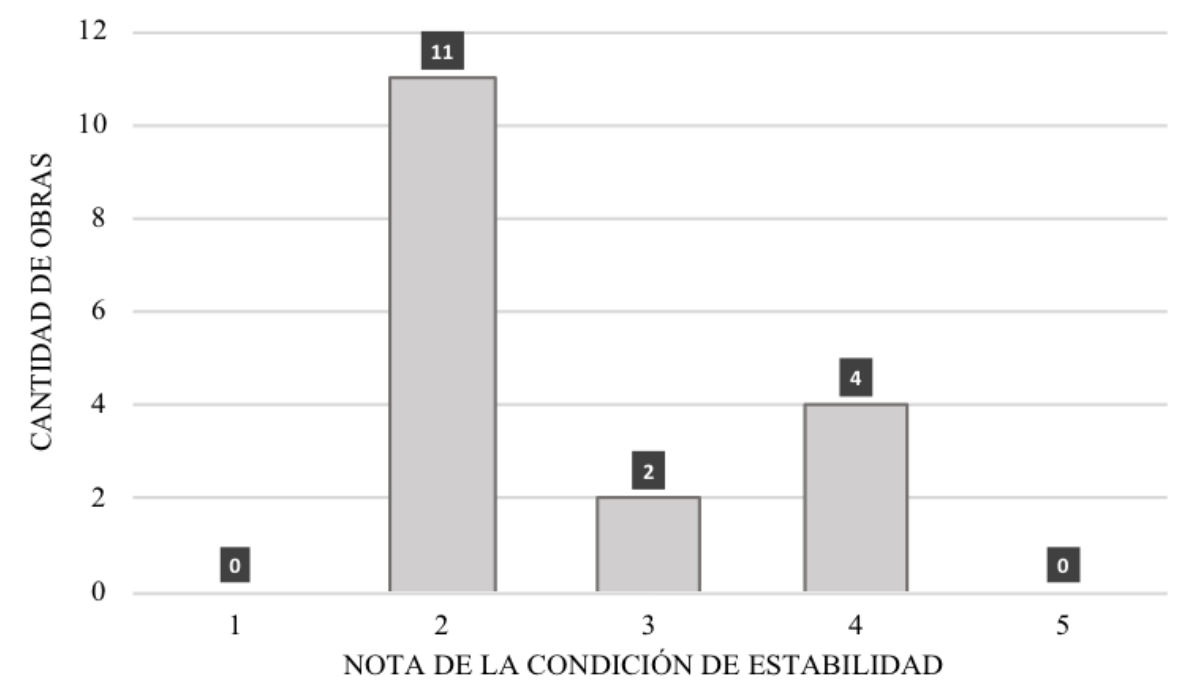

Figura 7. Análisis grafica de los reportes técnicos en los elementos estructurales de las OAE's que poseen daño por la acción biológica

La gran cantidad de obras que tienen un mal estado puede explicarse de acuerdo con NBR 9452 (ABNT, 2016). Según la literatura, el aumento del daño, junto con la presencia de humedad y el período de exposición, tienen una gran influencia en la desintegración de la cubierta de refuerzo. Así, considerando el elemento con anomalías en armaduras y hormigón, por Tabla E.2.9452 (ABNT, 2016), la calificación asignada está justificada.

\subsection{Estudio de caso}

Para este estudio de caso, analizamos la estructura: Puente sobre el río Bom Jesus, ubicado en el km 47.00. Los elementos componentes del puente se muestran en la Tabla 4, así como sus notas respectivas.

Tabla 4. Elementos estructurales del Puente y estado reportado

\begin{tabular}{c|c}
\hline Elementos Estructurales & Notas \\
\hline Losa de concreto armado & 3 - En observación \\
\hline Viga T o I en concreto armado & 2 - Problemática \\
\hline Transversa portante en concreto armado & 2 - Problemática \\
\hline Cortina de concreto armado & 3 - En observación \\
\hline Encuentro de albañearía con piedra & 5 - Excelente \\
\hline Aparato de soporte de péndulo & $5-$ Excelente \\
\hline Pavimento asfáltico & $5-$ Excelente \\
\hline Barreras rígidas tipo New Jersey & $5-$ Excelente \\
\hline Guarda cuerpo en concreto armado & $3-$ En observación \\
\hline Lleno de acceso & $5-$ Excelente \\
\hline Junta de dilatación & $4-$ Buena \\
\hline
\end{tabular}

Como se puede ver en la Tabla 4, la viga $\mathrm{T}$ o I y los elementos transversales de soporte son aquellos que muestran la evolución del daño por excrementos de murciélago y, por lo tanto, tienen una alta tasa de deterioro, como se puede identificar en la Figura 8. 


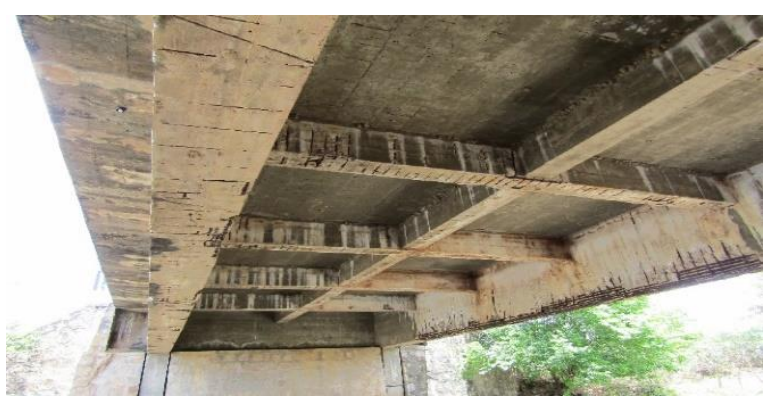

(a)

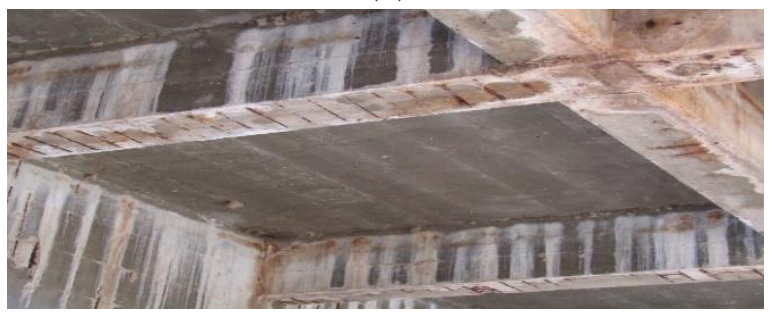

(c)

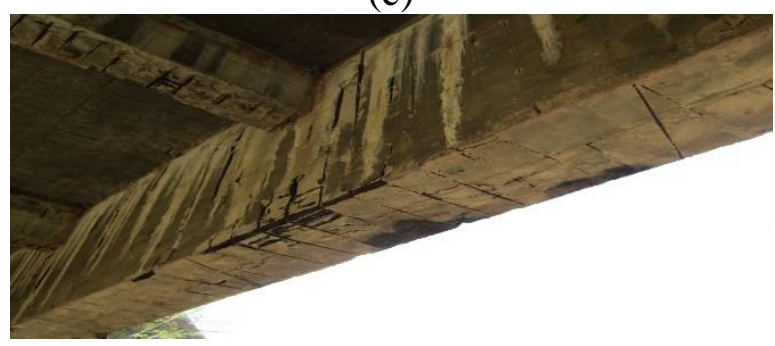

(e)

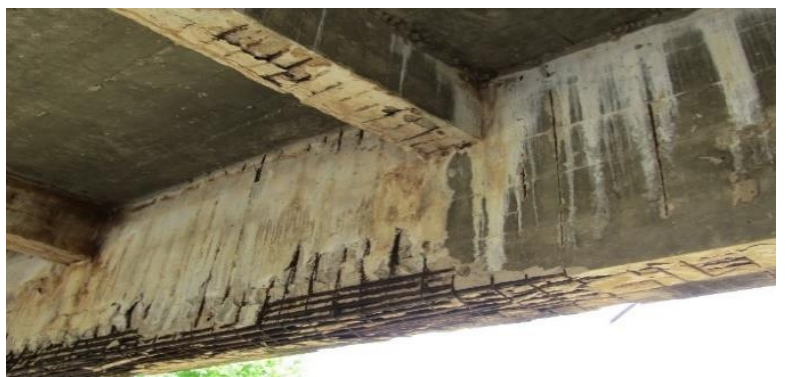

(b)

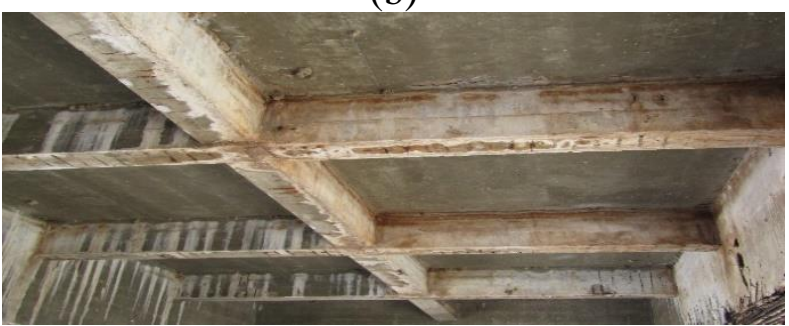

(d)

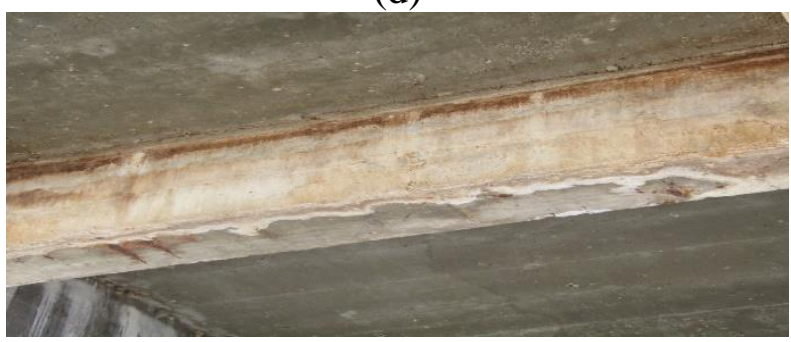

(f)

Figura 8. Reporte fotográfico del Puente sobre el Rio Bom Jesus

En estos registros fotográficos (Figura 9) fue posible verificar diferentes tipos de manifestaciones patológicas, causadas por varios factores y agentes de degradación. Entre las manifestaciones patológicas identificadas, se puede ver que la presencia de murciélagos aumentó la tasa de degradación en las vigas, produciendo daños como lixiviación y manchas de carbonatación, infiltración en el concreto, manchas de humedad, losas de concreto con refuerzo expuesto.

Las manchas causadas por el guano de murciélago no generan mucha preocupación a corto plazo, sin embargo, si no se someten a un seguimiento adecuado e intervenciones posteriores por un proceso de rehabilitación, pueden conducir al desarrollo de manifestaciones patológicas cada vez más graves.

Las manifestaciones patológicas que pueden deberse al guano de los murciélagos son: manchas de lixiviación y carbonatación, infiltración en concreto, manchas de humedad, concreto desnudo con refuerzo expuesto, armadura principal muy oxidada en puntos localizados, viga con ligera corrosión cuando Es una viga metálica dentro del proceso de reducciones.

Todas las patologías mencionadas anteriormente tienen una gran influencia en la cobertura necesaria. NBR 6118 (ABNT, 2014) proporciona una cobertura mínima para estructuras de hormigón armado, y este daño, junto con la degradación originada de los murciélagos, proporciona oxidación en el refuerzo, expansión y desprendimiento del hormigón.

Con respecto a la corrosión del refuerzo, los murciélagos contribuyen con soluciones ácidas que favorecen la despasivación del refuerzo y la reacción de oxidación. Las manchas de carbonatación desequilibran el pH del hormigón, dejando vulnerable el refuerzo.

Y, por último, la mancha de humedad / infiltración en el concreto, por ejemplo, causada por una deficiencia de los desagües hace que el agua se filtre en las estructuras, y cuando esta agua entra en contacto con el ácido guano (a través de la porosidad del concreto) puede desencadenar manifestaciones patológicas que pueden contribuir a otras patologías, e incluso pueden colapsar elementos estructurales. 


\subsubsection{Acciones externas generados de daños}

Todas las manifestaciones patológicas se desencadenan en cuanto a la acción de agentes externos que influyen en la aparición de diversos daños. Dichos agentes nocivos para las estructuras reaccionan con la superficie en contacto dando como resultado la degradación de estos elementos. Los ejemplos que se pueden ver de estos agentes externos son el agua misma de las infiltraciones, diversas filtraciones que con el tiempo deterioran la superficie. Otro ejemplo sería la presencia de excrementos de animales, especialmente los abordados en este estudio de caso, de los murciélagos, que causan una degradación en el concreto debido a esta acción biológica.

Las causas de la acción de estos agentes externos tienen como consecuencia la aparición de daños que también deben registrarse en los elementos vinculados, que son: mancha de lixiviación y carbonatación, infiltración en el concreto, mancha de humedad, desplazamiento del concreto con refuerzo expuesto, armadura muy principal. oxidado en puntos localizados, viga con ligera corrosión cuando se trata con una viga metalica dentro del proceso de oxidación.

El registro de estas condiciones y daños derivados de la degradación debida a una acción biológica siempre debe registrarse y permitir el monitoreo adecuado de estas influencias externas

\section{CONSIDERACIONES FINALES}

Las Obras de Arte Especiales (OAE's) son estructuras conocidas como puentes, viaductos y pasos peatonales. Se consideran obras expresivas de gran interferencia, ya que actúan directamente sobre el flujo del tráfico. Debido a su funcionalidad, los OAE's pueden convertirse en puntos críticos en una carretera cuando sus condiciones de uso no son favorables. En este sentido, las inspecciones de las OAE's son importantes para monitorear la degradación de las estructuras durante la vida del proyecto, para que la funcionalidad de la red de carreteras no se vea afectada. Por lo tanto, a partir de las diferentes acciones que degradan las estructuras, este estudio informó específicamente la aparición de degradación en las estructuras a través de acciones biológicas de los guanos de murciélago. La sección elegida para este análisis se encuentra en BR-226, en la Unidad Federativa de Rio Grande do Norte.

Fue posible identificar que el $40 \%$ de las OAE's de la sección analizada tienen manifestaciones de degradación y se verificó que estas obras tienen el mismo sistema estructural, es decir, la viga de hormigón es el elemento principal y dominante, que difiere de estructuras de losas de hormigón armado, que no muestran manifestaciones de estos mamíferos.

Otro punto indicado fue la nota de los elementos estructurales de las inspecciones realizadas por DNIT. Aproximadamente el 65\% de las estructuras encontradas con la presencia de murciélagos tienen un grado de 2 o menos, lo que lo hace expresivo dentro de la muestra disponible. Esto puede influir significativamente en la seguridad del vial en la carretera y la aceleración de la vida útil del componente de la estructura.

En análisis, El Puente sobre el río Bom Jesus, ubicado en el km 47.00, presenta una degradación crítica de los elementos estructurales debido a la acción de los guanos de los murciélagos. Se observó corrosión en el refuerzo y hormigón desagregado a gran escala, verificando condiciones problemáticas de conservación y estabilidad estructural para los elementos. Por lo tanto, para este trabajo específico, se sugiere elaborar un proyecto de rehabilitación de las áreas afectadas para evitar problemas futuros, con el objetivo de minimizar la aparición de organismos vivos en las obras, monitorear la proliferación y la influencia de los murciélagos en la vida útil de las estructuras.

Finalmente, el artículo demostró la importancia de identificar las degradaciones debidas a las diversas acciones, particularmente las biológicas, para mantener el funcionamiento adecuado de la OAE's dentro de un contexto vial. Considerando la adecuada prevención de este tipo de degradaciones, conjuntamente de un adecuado gerenciamiento de activos y tomando las medidas correctivas en el momento adecuado prolongaran la vida útil de la estructura a un bajo costo y generan un valor agregado en el uso del dinero público.

C. A. A. L. ANHAIA, et al 


\section{AGRADECIMIENTOS}

Los autores agradecen al Departamento Nacional de Infraestructura de Transporte (DNIT) por su apoyo en este trabajo.

\section{REFERENCIAS}

Angelo, A. M. V. (2004), "Análise das patologias das estruturas em concreto armado do Estádio Magalhães Pinto - Mineirão". Dissertação de Mestrado em Engenharia de Estruturas, Departamento de Engenharia Civil, Universidade Federal de Minas Gerais, Belo Horizonte, MG, p. 439.

Araújo, C. J. R. V. (2014), Vistoriando obras de arte especiais. Notícias da Construção, (1): 60-62. Associação Brasileira de Normas Técnicas (2014). NBR 6118: Projeto de Estruturas de Concreto Procedimento. Rio de Janeiro.

Associação Brasileira de Normas Técnicas (2016). NBR 9452: Inspeção de pontes, viadutos e passarelas de concreto - Procedimento. Rio de Janeiro.

Bastos, H. C. N.; Miranda, M. Z. (2017), Principais patologias em estruturas de concreto de pontes e viadutos: Manuseio e manutenção das obras de arte especiais. Construindo, Belo Horizonte, 9:93-101. Bredt, A.; Uieda, W.; Pinto, P. P. (2009), Visitas de morcegos fitófagos a Muntingia calabura L. Muntingiaceae em Brasília, Centro-oeste do Brasil. Revista Brasileira de Zoociências, 4(1).

Departamento Nacional de Infraestrutura de Transportes (2004). DNIT 010: Inspeções em Pontes e Viadutos de Concreto Armado e protendido - Procedimento. Rio de Janeiro.

Fonseca, R. P. (2007) "A estrutura do Instituto Central de Ciências: Aspectos históricos, científicos e tecnológicos de projeto, execução, intervenções e propostas de manutenção." Dissertação de Mestrado em Estruturas e Construção Civil, Departamento de Engenharia Civil e Ambiental, Universidade de Brasília, Brasília, DF, 213 p.

Horta, E. F. (2000), Sistemas de gerenciamento de obras de arte. Téchne, São Paulo, p.1-7

Lima, M. G. (2005). Ação do meio ambiente sobre as Estruturas de Concreto. ISAIA, G. Concreto: Ensino, Pesquisa e Realizações, IBRACON, São Paulo, (1):713-751.

Medeiros, M. H. F.; Andrade, J. J. O.; Helene, P. (2011), Durabilidade e Vida Útil das Estruturas de Concreto. Concreto: Ciência e Tecnologia. Ed. G. C. ISAIA. Instituto Brasileiro do Concreto: IBRACON, São Paulo, 1(1).

Pacheco, S. M., Sodré, M., Gama, A. R. Bredt, A., Cavallini, E. M., Marques, R. V., Bianconi, G. (2010), Lista anotada dos mamíferos do Brasil/Annotated checklist of Brazilian mammals. Occasional Papers in Conservation Biology, 16(1):629-647.

Reis, N. R. (2007). "Morcegos do Brasil". Londrina, Paraná, 253p.

Siedel, H.; Leisen, E. V. P.; Leisen, H. (2008). "Salt load and deterioration of sandstone at the temple of angkor wat, Cambodia." 11 International Congress on Deterioration and Conservation of Stone, Torun, Poland. Proceedings, v. 1, pp.267-274.

Steiger, V. K. (2015) "Contribuição para o estudo da degradação química da pasta de cimento: Simulação da atividade agressiva do guano de morcegos." Dissertação de Mestrado em Engenharia e Tecnologia de materiais, Departamento de Engenharia Civil, Pontifícia Universidade Católica do Rio Grande do Sul, Porto Alegre, RS, 118 p.

Vitório, A. (2003) "Fundamentos da patologia das estruturas nas perícias de engenharia". Instituto Pernambucano de Avaliações e Perícias de Engenharia. Recife, 58p.

Wei, S., Jiang, Z., Liu, H., Zhou, D., Sanchez-Silva, M. (2013), Microbiologically Induced deterioration of Concrete - A Review. Brazilian Journal of Microbiology. 44(4):1001-1007. http://dx.doi.org/10.1590/S1517-83822014005000006 